\title{
A PROPÓSITO DEL ESQUEMA DE FINANCIAMIENTO DE LA UNIVERSIDAD PÚBLICA
}

\section{Daniel Libreros Caicedo Universidad Pedagógica Nacional}

Uno de los temas más discutidos durante el período previo a la aprobación del Plan de Desarrollo, fue el de la educación superior, particularmente en lo que se relaciona con la propuesta de modificar la forma actual de asignación de recursos a las universidades públicas. Se trataba de oficializar el sistema de pago por subsidio a la demanda (crédito educativo focalizado), el cual las obligaría a competir —en condiciones desiguales - con las universidades privadas y a elevar de manera considerable el monto de las matrículas. Esta propuesta, que se inscribe en línea de continuidad con lo que los organismos internacionales de crédito (caso Fondo Monetario Internacional) vienen exigiendo en la región, no fue incluida en la versión final del Plan aprobada por el Congreso, gracias a la presión de los rectores y las comunidades educativas de las universidades oficiales; sin embargo, es un hecho, que esta discusión no ha terminado y que asistiremos en los próximos meses a la continuidad de este debate en otros espacios.

\section{La crisis económica actual}

El diagnóstico del Plan de Desarrollo reconoce en estadísticas los malos resultados económicos que el país viene evidenciando en los últimos años: caída en el crecimiento anual del producto interno bruto (PIB), balanza de pagos (relación entre exportaciones e importaciones) negativa, déficit fiscal y aumento del desempleo, entre otros. Sin embargo, se queda corto en las cifras; así, en las dos versiones oficiales del Plan, la presentada inicialmente por el gobierno y la que se presentó ante el Congreso de la República con el aval del Consejo Nacional de Planeación, se estimó en $1.8 \%$ la tasa de crecimiento del PIB para 1998. No obstante, esa cifra era irreal: se ratificó el estado de recesión que atraviesa el país, se constató una tasa de crecimiento del PIB de apenas $0.2 \%$, la más baja desde 1942 , la cual explica, a su vez, una tasa de desempleo cercana al $20 \%$, la peor de las últimas décadas.

En marzo de 1999 , el conjunto de la industria arrojó pérdidas por $\$ 400.000$ millones (un año atrás, había reportado utilidades trimestrales de $\$ 245.000$ millones). El consumo de energía, indicativo del ritmo de actividad económica, registró una variación negativa en enero de este año, continuando la tendencia a la baja que trae desde enero de 1998. La variación anual acumulada de la producción industrial de bienes de consumo fue 1\% en febrero de 1999 (casi cinco puntos por debajo de lo registrado apenas un semestre atrás), la de bienes intermedios fue $-2 \%$ (en agosto de 1998 había sido $3.8 \%$ ), y la de bienes de capital fue $3.8 \%$ (cuando seis meses antes había sido 3.8\%).

Adicionalmente, la calidad de la cartera del sistema financiero (cartera vencida! cartera bruta) alcanzó el $12.1 \%$ a finales de febrero de 1999; para los bancos, sin Caja Agraria, el indicador presentó un valor $10.8 \%$, y para las corporaciones de ahorro y vivienda un preocupante $14.8 \%$, que hoy las sitúa a la cabeza de la lista de las instituciones financieras con mayores problemas de liquidez. Ello explica el aumento de los bienes dados en pago 
como porcentaje del patrimonio, que reflejan la evolución de las garantías prendarias en créditos hipotecarios, el cual subió desde 12.5 puntos en junio hasta 22.5 en diciembre del mismo año, en el caso de las corporaciones de ahorro y vivienda.

Y, por si hacen falta más luces rojas, debe tenerse en cuenta que entre 1997 y 1998 el país registró 200 concordatos, y que desde 1996 se liquidaron 205 empresas, de las cuales 91, tan sólo en 1998.

Para acompañar ese panorama desolador, basta apenas con mencionar la situación de la economía mundial, que tampoco fue bien calculada en las proyecciones contenidas en los documentos gubernamentales. Hoy no se descarta una merma en el ritmo de crecimiento de las economías desarrolladas, el cual podría derivar en una espiral recesiva en el propio grupo de los siete. Las economías del sudeste asiático aún no se recuperan de la "catástrofe económica" de 1997; Brasil continúa en una coyuntura crítica; Ecuador sucumbió ante una crisis financiera y bancaria sin precedentes, y Venezuela campea la crisis a la espera de reformas políticas y económicas importantes ${ }^{29}$. La situación de estos dos últimos países viene repercutiendo contra la integración andina; la ruptura de los convenios, en lo atinente al transporte de carga pesada por parte de Venezuela y la discusión con Ecuador sobre el asunto de la importación de arroz, está confirmando que en épocas de crisis las necesidades de cada país pasan por encima de acuerdos anteriores y de compromisos de unidad regional.

Además, las estadísticas de asesinatos individuales y colectivos siguen confirmando a Colombia como uno de los países con más asesinatos en el mundo (aproximadamente 30.000 al año), asesinatos que, en gran medida, quedan impunes. Para terminar esta enumeración de elementos negativos, debe añadirse que compartimos con Brasil el indicador Gini de pobreza más alto de Latinoamérica y uno de los mas altos en promedios internacionales.

Pero el Plan de Desarrollo no sólo se queda corto en las estadísticas que confirman la crisis, sino que llega a la conclusión -que actualmente se discute hasta en el equipo de dirección del Banco Mundial- de que lo que explica este escenario adverso es el no haber llevado a fondo las políticas de ajuste fiscal. Plantea que en el futuro inmediato se debe reducir aún más el gasto público, mejorando la gestión eficiente de los recursos:

Posteriormente los últimos años vieron cómo se trató de reorientar la acción del Estado para, en vez de homogenizar y profundizar esas reformas, tratar de enfatizar su contenido social dándoles un sentido más asistencialista. Este esfuerzo de reorientación se concentró sobre la acción del Estado, más que sobre su organización y procedimientos... ${ }^{30}$.

La negación del asistencialismo implica un nuevo pacto participativo, el cual debe basarse en la distribución de responsabilidades a la comunidad, antes que en la asignación de presupuestos: "La participación no será ya alrededor del reparto de unos recursos del gobierno central, sino en tomo a la distribución de responsabilidades y a la consolidación de

\footnotetext{
29 Estos datos han sido tomados de "Breves comentarios al Plan Nacional de Desarrollo $1999-2002$ Borrador para análisis y discusión”, Oliver Mora Toscano y Carlos Betancurt, ESAP, Programa de Finanzas Públicas —Minhacienda- PNUD, 1999, inédito.

${ }^{30}$ Plan Nacional de Desarrollo 1998-2002- “Cambio Para Construir la Paz”, D.N.P, Bogotá-1999, p.6. 
recursos locales para solucionar los problemas de las comunidades..."31. Además, para estabilizar este tipo de participación se requiere recuperar la legitimidad institucional y nuevos consensos ciudadanos. El proceso de paz aparece entonces, para los voceros gubernamentales, como el escenario en el que se deben conseguir estas metas; la paz se convierte en una necesidad imperiosa para estabilizar las políticas de ajuste, para que la sociedad produzca "capital social" 32 .

Y si las definiciones sobre el ajuste atraviesan conceptualmente hasta la democracia participativa y la negociación política con los grupos armados, igualmente lo hacen con la política social en general y con la política educativa en particular ${ }^{33}$. La asignación fiscal, conforme a subsidio a la demanda, persigue una reducción importante del gasto educativo al desconocer el pago conforme a los costos históricos de las nóminas de los docentes reconocido en la Ley 60 de 1993 (el 24,5\% de los ingresos corrientes); el crédito focalizado a las universidades públicas intenta lo propio en el área de la educación superior.

La "concepción de sociedad" que se esconde en este tipo de fórmulas va mucho más allá de lo económico; se trata de "mercantilizar" la política social. La mercantilización de los servicios viene presentándose por los técnicos gubernamentales como el nuevo paradigma en la ejecución de los mismos ${ }^{34}$. El papel del Estado debe ser el de garantizar que el "mercado social" funcione, incluso en los niveles del SJSBEN (Sistema de información de necesidades básicas insatisfechas); se focaliza el gasto para que los pobres accedan al mercado. El Estado perdió la definición de la política social, quedando reducido al papel secundario de manejar las limitaciones ("externalidades") del "mercado de los bienes públicos" y a garantizar los negocios que puedan operar en el mismo.

El debate acerca del diseño de los indicadores de gestión, contra los que se reconocerá igualmente transferencia fiscal, se ubica en este contexto. Una concepción mercantilizada de la educación superior intentará reducirlos a una simple relación cuántica costo-beneficio inmediato, desconociendo el papel de las universidades en temas sociales complejos como la creación de espacios de tolerancia, la participación directa en los procesos de pacificación, el aporte de la formación a las responsabilidades ciudadanas, por citar ejemplos notorios. Esta es la razón del porqué los rectores de las universidades públicas continúan exigiendo en

\footnotetext{
${ }^{31}$ bid., p. 29.

${ }^{32}$ Citan la definición de Stiglitz, uno de los voceros más importantes del B.M. durante los últimos años sobre el tema: "el pegamento que produce cohesión entre y al interior de los grupos". Ibíd., p 27.

${ }^{33}$ Estas políticas de ajuste se explican por las exigencias del pago de la deuda externa. En la actual coyuntura, el tema de la deuda ha recobrado particular importancia. La devaluación que se viene generalizando en el continente, como producto de la devaluación brasileña —que representa el 53\% de la economía regional—, viene produciendo un aumento a escala de las deudas externas públicas de tal magnitud que asfixia el gasto público. En el caso colombiano, sumando la de deuda de corto y largo plazo, ya llega a $\$ 17,5$ billones. Debe tenerse en cuenta que el propio gobierno anunció una devaluación de $25 \%$, para lo que quedaba de 1999 , proyección que a nuestro entender se queda corta dada la magnitud de la crisis económica internacional. De llegar a cumplirse el pronóstico presidencial, el incremento de la deuda pública, por ese sólo concepto, sería de $\$ 5,1$ billones. Además, el FMI, tal y como lo hizo en la década del 80 , presionará a los gobiernos para que asuman parte del endeudamiento privado ( $\$ 16$ billones entre deuda de corto y largo plazo) por diversos mecanismos: diferencial cambiario, operaciones de salvamento, compra de títulos-deuda, etc. Luis Guillermo Vélez, presidente de la Comisión económica del Senado, ya se pronunció a favor de la reestructuración del $50 \%$ de la deuda privada. Ver entrevista: "El Espectador", sección salmón, p. 4-B, abril 4 de 1999.

34 Al respecto, ver "Informe Wiesner-Bird, DNP, Bogotá, 1991: "La efectividad de las políticas públicas en Colombia”; y Eduardo Wiesner, Tercer Mundo Editores, Bogotá, 1998. 
la actualidad que esos indicadores sean concertados entre gobierno y comunidades universitarias.

\section{El diagnóstico neoliberal de la universidad pública}

La justificación teórica de la política de financiamiento para la universidad oficial que alcanzó a anunciarse durante la discusión pública del Plan de Desarrollo se ha presentado desde hace varios años en comisiones gubernamentales; un buen resumen de esta justificación lo constituye el diagnóstico realizado por Jesús Duarte y Carlos Villa desde $1997^{35}$, quienes han desempeñado cargos de responsabilidad administrativa en el sector. Para estos autores las deficiencias de la universidad pública se resumen en:

- El gasto público promedio en educación superior durante los últimos años (período 90-96) se ha incrementado sin que aumente su "eficiencia" (del 0,57 al 0,77\% del PIB). Debe añadirse que esta cifra no es alta comparada con otros países de América Latina, para no hablar del caso de los países industrializados, como tampoco ha significado un incremento considerable para el período comparado con otros rubros caso endeudamiento público (el cual en el año 96 se acercaba a 8 puntos del PIB y en la actualidad llega a casi el $14 \%$ del PIB). Los autores añaden en sus argumentos que el gasto público en educación superior favorece a los estratos altos $(4,5$ y 6$)$ lo cual, supuestamente, puede comprobarse por estadísticas, desconociendo el "filtro social" de la pobreza que también se puede demostrar por estadísticas, el aumento en el número de pobres absolutos en el país (cifra que ya se acerca al 55\% aproximado del total de la población). Esto es lo que explica la deserción escolar; de cada 1000 niños que ingresan al primer grado de la básica primaria tan sólo 1 llega a la educación superior, en el medio quedan rezagados los niños trabajadores, los niños informales, las adolescentes prostitutas y demás. No puede responsabilizarse a la universidad pública de un problema estructural de la sociedad colombiana.

- La diferencia en la obtención de recursos entre universidades privadas y públicas, incluyendo las territoriales. Aquí el argumento es de perogrullo, se anota que mientras las universidades privadas se financian con el pago de matrículas $(80 \%)$ y con la venta de servicios $(20 \%)$, en el caso de las universidades oficiales la proporción es inversa (tan sólo el $20 \%$ se obtienen por recursos propios). Obviamente que estos resultados reflejan los diferentes propósitos de estas instituciones. La razón de ser de las universidades privadas es el lucro, mientras que la responsabilidad de las universidades del Estado debe ser la de responder a las necesidades de formación profesional y de transmisión del conocimiento para los sectores populares. El argumento, además, esconde el hecho de que el incremento de la cobertura en las universidades privadas, en mucho ha sido conseguido por su transformación en "universidades de masa" ${ }^{36}$, instituciones que aumentan cober-

\footnotetext{
35 "Hacia un nuevo esquema de financiación de la universidad pública colombiana" ,Jesús Duarte y Carlos Villa. En: "Revista Colombiana de Educación", Plaza y Janés- CIUP de la Universidad Pedagógica Nacional, Santafé de Bogotá, primer semestre de 1997.

${ }^{36}$ El concepto es utilizado por Rodrigo Parra Sandoval en "La Calidad de la Educación, Universidad y cultura popular", Editorial Tercer Mundo, Bogotá 1992, para indicar que el decreto 80 de 1980 oficializó una demanda fragmentada de la educación superior por la vía de los centros tecnológicos y las universidades nocturnas mediante la cual las capas medias bajas y los sectores populares encontraron un nivel de formación profesional 
turas sin calidad, en detrimento de una formación integral y adecuada para actuar en el universo del trabajo de los profesionales.

— La estructura de los gastos. Mientras que las universidades públicas ejecutan en funcionamiento el $85 \%$ de los gastos, en las privadas este monto llega tan sólo al $50 \%$; obviamente que ello permite diferencias importantes en el manejo de los gastos de inversión, $15 \%$ promedio en las instituciones de educación superior del Estado y $25 \%$ promedio en las privadas. Esto, supuestamente explica los excesivos costos por estudiante en la universidad pública, costos que aumentaron del 1,86\% promedio durante la década del 80 al $6 \%$ aproximado en la del 90, lo cual sirve de marco al ya conocido alegato de que la responsabilidad de este incremento es el decreto 1444 de 1992 (decreto que oficializó los aumentos saláriales a los docentes por puntaje obtenido conforme a investigación y publicaciones), la planta excesiva de profesores y personal administrativo, la excesiva estabilidad y demás. El modelo a seguir debe ser, entonces, la informalización del trabajo docente, los contratos a término fijo que impiden la investigación seria ${ }^{37}$ un indicador que confirma esta tesis es la del número de doctores, $2,6 \%$ en el conjunto de la educación superior (cifra reconocida por los propios autores).

\section{La alternativa del mercado educativo}

La solución de los problemas de las universidades oficiales, para estos autores, es la de privatizarlas, dejando que las matrículas oscilen conforme a las reglas del mercado y mejorando la venta de servicios, al tiempo que se establece la política compensatoria y focalizada de créditos educativos a las poblaciones marginales. Estos créditos serían asignados por un fondo creado para el efecto con los dineros de las instituciones estatales que actualmente financian la educación superior estatal (caso ICETEX, COLCIENCIAS) y administrado bajo los criterios de la banca privada ${ }^{38}$. Los créditos educativos condicionarían entonces, la oferta de programas "pertinentes" quedando este criterio incluido en el Sistema Nacional de Acreditación. Las carreras con énfasis humanista quedarían prácticamente excluidas en este sistema mercantilizado de la educación superior, sesgando la formación hacia lo técnico-instrumental. Habría que añadir, ¿y qué profesión garantiza en el futuro inmediato el acceso al mercado laboral con la crisis económica que estamos soportando?

\section{Las verdaderas causas del problema}

La carencia de "criterios objetivos y transparentes" en la asignación del gasto, recurrente en los discursos oficiales, remite a un supuesto protagonismo excesivo del Estado; Duarte y Villa añaden que en gran parte el problema de las debilidades en la financiación de la

\footnotetext{
inferior al convencional y el cual sólo les permite acceder a las áreas peor pagas del mercado laboralprofesional.

${ }^{37} \mathrm{E} 1$ artículo citado reconoce que los recursos asignados por COLCIENCIAS para investigación en la educación superior pública para el año 1996 fue de $\$ 9.036$ millones, cifra que significó el $40 \%$ del monto total por ese mismo concepto. Haciendo la proyección se llega a una cifra aproximada de $\$ 20.000$ millones, cifra irrisoria para los propósitos de una investigación seria en el nivel más alto de la educación y conforme a las necesidades de la tan pregonada "sociedad del conocimiento".

${ }^{38}$ Al hacer esta propuesta se critica de paso la "ineficiencia" actual del ICETEX por baja cobertura, por cuanto, en 1995 sólo atendió el 35,7\% de las demandas en pregrado y el 95\% en postgrado, escondiendo el asunto de los recortes presupuéstales. Igualmente lo critican por mal administrador ya que su cartera vencida representó el $13,9 \%$ en 1995 y el $13,2 \%$ en 1996, añadiendo que se requiriere a futuro un régimen draconiano para garantizar el retorno de los dineros.
}

Digitalizado por RED ACADEMICA 
educación superior obedece a la negociación de los presupuestos entre los diversos agentes estatales. Esta caracterización está tomada de Albrecht y Ziderman ${ }^{39}$, quienes han planteado desde el Banco Mundial que ésta es una de las formas-tipo de distribución de los presupuestos actualmente en la mayoría de los países en desarrollo. Ello por cuanto el aumento considerable de las presiones sociales en esta área educativa en las últimas décadas, dada la expansión de las matrículas en primaria y secundaria, las exigencias formativas del trabajo especializado y la percepción social de que "el acceso a la educación superior permite la prosperidad individual y mejora la equidad", ha desbordado las capacidades presupuéstales de los Estados. Entre 1960 y 1987, las matrículas en educación superior, para el caso de los países en desarrollo, se incrementaron por cuatro en los de bajos ingresos, por diez en los de ingreso medio y por nueve en los de ingreso alto. En el caso de América Latina, las estadísticas constatan que en el período 1975-1985, las matrículas en educación superior crecieron en un $370 \%$ mientras que el gasto público del sector lo hizo en $210 \%$. De esta manera, el gasto público por estudiante cayó en $34 \%{ }^{40}$ Para reducir la "demanda" los gobiernos implementaron restricciones como exámenes de Estado para el ingreso a la universidad, controles financieros en las transferencias por costo estudiante o por costo planta docente y controles a la ejecución interna del gasto dentro de las instituciones.

A este reconocimiento de los consultores del Banco Mundial debe añadirse que la reducción deliberada del gasto fiscal en servicios en general y en la educación superior en particular, como práctica gubernamental, acompañó la reforma política neoliberal en el continente, reforma que liquidó al viejo Estado patrimonialista, el cual, a pesar de su carácter corporativo-rentista, de control privado a las decisiones estatales, cumplía funciones de "Estado de Bienestar" 41 . Esta reforma, como se ha anotado anteriormente, estuvo en gran medida condicionada por los organismos internacionales de crédito, a raíz de la declaratoria de la moratoria en los pagos de la deuda externa oficializada por los países más importantes del continente a mediados de la década del $80^{42}$.

\footnotetext{
${ }^{39}$ Albrecht, D. y Ziderman, A. "Funding Mechanisms for Higher Education”. Banco Mundial

40 Ibíd. P. 3, los cuales a su vez toman el dato de Winkler - 1990, estudio sobre Argentina, Brasil, Chile, Colombia, México y Venezuela.

${ }^{41}$ Las razones por las cuales el Estado en Colombia y América Latina, previo a la reforma neoliberal, puede caracterizarse como "Estado de Bienestar" son:

-Decidida intervención en el proceso productivo para asumir un conjunto de funciones necesarias a la ampliación del proceso de acumulación (inversiones en infraestructura física y social; financiación de las mismas mediante políticas fiscales y monetarias; montaje de empresas estatales en sectores estratégicos productivos).
}

-Manejo de la política monetaria y fiscal con carácter anticíclico, promoción de inversión en la recesión y de políticas contraccionistas en épocas de expansión.

-Papel decisivo en la reproducción de la fuerza de trabajo vinculada a actividades productivas y estatales fundamentales. Instituciones y programas públicos en instituciones técnicas, subsidios al transporte, la alimentación y la salud, recreación, vivienda y educación, etc.

-Instituciones estatales reguladoras de los servicios públicos y sociales, y de la propia inversión privada.

-Promoción de la concertación entre los diversos agentes productivos, mediante una legislación laboral que regula los procesos del trabajo, construyendo derechos sociales. Tomado de Édgar Novoa y Darío Restrepo en "Las políticas sociales de la Nueva Era", SINAP-ESAP, enero de 1994.

${ }^{42}$ En el caso colombiano, esta tesis es igualmente válida aun cuando el problema del endeudamiento externo, tanto público como privado, no llegó a conocer las dimensiones de los países que durante el período habían apostado a la reconversión industrial; particularmente durante el gobierno de Betancur se presentó una escasez de divisas que llegó a colocar en riesgo el pago de los endeudamientos. La descentralización fiscal como mecanismo de reducción del gasto, impuesto por el FMI, iniciará el camino de la "modernización del Estado" 
La mercantilización de lo social se enmarca en ese contexto. Pero, a medida que pasan los años, las propuestas se perfeccionan. El Plan de Desarrollo ha abierto mayores posibilidades a esa mercantilización por la vía de la intermediación financiera; sistemas de crédito con titularización de matrículas y de activos de las instituciones educativas se vienen abriendo paso en la educación superior, a la manera de lo que ocurrió con las pensiones o con la ejecución del gasto en salud en los años anteriores. No se trata tan sólo de recortar presupuestos, sino que además se pretende generalizar el endeudamiento en el "sistema de la educación superior" con aval gubernamental. Razón adicional para que el "escaso" 10\% que por concepto de matrículas cubre la totalidad de los costos en la educación superior pública sea reconsiderado; por cada punto adicional que aumenten los costos de las matrículas, se incrementaría el negocio de la titularización-crédito. No importa que cada vez se reduzcan las posibilidades de acceso a las universidades oficiales, lo importante es que el negocio funcione. Es uno de los signos de la época: la democracia limitada a las posibilidades del mercado. A ello debemos responder las comunidades educativas de las universidades públicas.

que se consolidará con la reforma constitucional de 1991. Haciendo una alusión al contenido de estas reformas institucionales Édgar González Salas, actual director del departamento de Función Pública, anota: "La reducción del déficit fiscal, como instrumento de política esencial para controlar los desequilibrios macroeconómicos como la inflación y el desajuste del sector externo, así como para permitir la canalización de los recursos indispensables para el pago de la deuda externa, tiene en este escenario una importante conexión con la reforma tributaria en sus aspectos sustanciales y procésales - de índole administrativa-, con la expedición del nuevo Estatuto Orgánico de Presupuesto y con la descentralización administrativa y fiscal ahondada con la expedición de los estatutos de 1987. En una palabra con importantes frentes de acción de la Administración Pública...". En: "El Laberinto Institucional Colombiano 1974-1994

—Fundamentos de Administración Pública”, Universidad Nacional-FESCOL_ESAP, 1997, p. 206. 